\title{
Proximity-activated nanoparticles: in vitro performance of specific structural modification by enzymatic cleavage
}

\author{
R Adam Smith \\ Sarah L Sewell \\ Todd D Giorgio \\ Department of Biomedical \\ Engineering, Vanderbilt University, \\ Nashville, TN, USA
}

Correspondence:Todd D Giorgio

Vanderbilt University, Box 351620, Station

B, Nashville, TN 37235, USA

Tel +l 6153223756

Fax +I 6153437919

Email todd.d.giorgio@vanderbilt.edu

\begin{abstract}
The development and in vitro performance of a modular nanoscale system capable of specific structural modification by enzymatic activity is described in this work. Due to its small physical size and adaptable characteristics, this system has the potential for utilization in targeted delivery systems and biosensing. Nanoparticle probes were synthesized containing two distinct fluorescent species including a quantum dot base particle and fluorescently labeled cleavable peptide substrate. Activity of these probes was monitored by gel electrophoresis with quantitative cleavage measurements made by fluorometric analysis. The model proximityactivated nanoparticles studied here exhibit significant susceptibility to cleavage by matrix metalloprotease-7 (MMP-7) at physiologically relevant concentrations, with nearly complete cleavage of available substrate molecules after 24 hours. This response is specific to MMP-7 enzyme activity, as cleavage is completely inhibited with the addition of EDTA. Utilization of enzyme-specific modification is a sensitive approach with broad applications for targeted therapeutics and biosensing. The versatility of this nanoparticle system is highlighted in its modular design, as it has the capability to integrate characteristics for detection, biosensing, targeting, and payload delivery into a single, multifunctional nanoparticle structure.
\end{abstract}

Keywords: quantum dot, MMP-7, protease, proximity activated nanoparticle

\section{Introduction}

Localized or specific delivery of therapeutics to diseased cells represents a historic paradigm shift in the treatment approach toward many disease states, including various types of cancer. Traditional approaches utilize systemic administration of highly potent drugs in an attempt to eradicate neoplastic cells, often with significant toxic side effects. Initial attempts at targeted delivery of these drugs have focused on the incorporation of small ligands to direct the therapy to a specific target site (Sachdeva 1998; Hallahan et al 2001; Pastorino et al 2003; Mitra et al 2005). However, the current generation of targeted therapies has not yet generally achieved highly specific localization to tumors and emerging neoplasia through a single recognition mechanism (Bogenrieder and Herlyn 2003; Kim and Toge 2004). Nonspecific binding and specific binding to nontarget cells compromise the therapeutic index of small molecule, ubiquitous cancer targeting ligands, and limits their applicability and efficiency as primary chemotherapeutic agents.

An alternative targeting approach involves the use of molecules capable of activation following cleavage by tumor- or other tissue-specific enzymes. These compounds have been incorporated into many structures ranging from in vivo imaging constructs (Weissleder et al 1999; Tung et al 2000; McIntyre and Matrisian 2003; Harris et al 2006 ) to chemically modified drug structures in the form of prodrugs (Suzawa et al 2000, 2002; Liu et al 2003). These studies demonstrate the feasibility of tumor-specific 
enzyme interaction as a means of modifying the physical structure of delivered drugs and imaging constructs, and potentially enables targeted delivery of drug carriers, including nanoparticles (NPs).

Several studies utilizing this approach have focused on the development of tumor-specific molecular imaging constructs cleavable by matrix metalloproteases (MMPs), including specific cleavable peptide sequences (Bremer et al 2001, 2002; Zucker and Cao 2001; McIntyre and Matrisian 2003; McIntyre et al 2004). The basis of the MMP-7 specific peptide substrate (RPLALWRS) utilized in one such report (McIntyre et al 2004) is employed in this study to impart tumor-specific activity to our NP proximity activated system.

\section{The role of MMP-7 in biological processes}

MMPs (including MMP-7) are a family of extracellular, zinc-dependent proteases that have a role in tissue breakdown and remodeling during both normal (eg, angiogenesis, gut innate immunity) and pathological (eg, inflammation, tumorigenesis, metastasis) processes. MMP-7 is important in the progression of a number of tumors, notably those originating in the colon or breast (McIntyre et al 2004). Furthermore, MMP-7 has proven to have diagnostic and prognostic value, as expression is associated with poor outcome in esophageal (Yamamoto et al 1999), colon (Adachi et al 2001), and pancreatic (Yamamoto et al 2001) cancers.

For tumor systems in which MMP-7 activity is known to modulate the course of the disease, MMP-7 is secreted even in the earliest stages. Small, benign "precancerous" lesions, for example, exhibit MMP-7 secretion well before these lesions would be clinically detectable (Crawford et al 2002; Hulboy et al 2004). Furthermore, analysis of MMP-7 expression in human neoplasia revealed that enzyme mRNA appeared in the surrounding normal tissue in only a few isolated cases (Wilson and Matrisian 1996), suggesting highly specific enzyme expression in the immediate tumor vicinity.

\section{Nanoparticle-based therapeutic systems}

Literature citations of tumor-specific cleavable constructs focus mainly on molecular imaging of enzyme activity and drug activation in vivo. However, incorporation of these constructs into NPs or their application to target NP-based therapeutic systems is an exciting area of opportunity. One recent study (Zhang et al 2006) has incorporated cleavable
MMP substrates into NPs via a biotin-streptavidin linkage, outlining one method utilizing specific enzymatic activity to modulate NP uptake and delivery to cells. The work described here offers a conjugation approach that eliminates the need for biotin-streptavidin, resulting in a multifunctional nanoscale construct with novel characteristics. Importantly, studies described here include quantitative examination of NP construct cleavage and activation, providing a robust basis for further in vitro and in vivo targeting, delivery, and sensing applications.

Particulate systems have inherent advantages for these applications, in part, due to their relatively large surface to volume ratio, allowing the conjugation of multiple active molecules directly to the particle surface. Stable covalent conjugation of a range of bioactive molecules such as enzymes (Phadtare et al 2004; Rossi et al 2004; Kuhn et al 2006, 2007), mAbs (Tang et al 2005), peptides (Liu et al 2001), and DNA (Wang et al 2003; Zhang et al 2004) to the surface of NPs has been reported. Direct conjugation of ligands to the NP surface may overcome significant obstacles inherent in the ubiquitous biotin-streptavidin system, including immunogenicity, increased molecular size (primarily due to the relatively large molecular mass of streptavidin), and limited in vivo application. With small molecule ligands, many functional copies can potentially be directly attached to the surface of the NP enabling multiplexed interaction with target cells. In addition to these attractive nanoparticle characteristics, quantum dots (QDs) offer the added benefit of intense fluorescence characteristics for highly sensitive detection and imaging. The combination of these attributes impart the capability to integrate specific delivery, detection, imaging, and treatment characteristics into a single, multifunctional NP delivery vehicle.

In this study, we describe the synthesis and in vitro performance of a NP probe capable of specific activation by MMP-7. In addition, the importance of quantitative analysis of construct performance is described in the context of in vitro and in vivo application. To construct the proximity activated NP, a carboxylated QD was functionalized with a peptide construct $\left(\mathrm{PEG}_{3400}\right.$-[Ahx]-RPLALWRS-[Ahx]$\mathrm{PEG}_{5000}-\mathrm{K}(5-\mathrm{FAM})$; PA construct). The PA construct consists of an 8-mer peptide substrate (RPLALWRS) cleavable by MMP-7. The PA construct also contains polyethylene glycol (PEG) groups to reduce nonspecific binding. Therefore, the PA construct initially presents as a PEGylated nanoparticle with low nonspecific binding as predicted from previous studies (Akerman et al 2002; Ballou et al 2004; Gao et al 2004). Cleavage of the internal peptide liberates the distal 
PEG and part of the peptide, along with a fluorophore, in this work, for assessment of cleavage (Figure 1). In the future, proteolytic modification could be used to "unmask" the particle, enabling access to underlying small molecule ligands previously concealed near the nanoparticle surface. Effective PA targeting will likely be modulated by many molecular factors, including the relative characteristics of the distal and proximal PEGs on the PA construct and the PEG (or other molecular spacer) that displays the ligand from the nanoparticle surface.

\section{Materials and methods Cleavable peptides and NP probes}

The PEGylated substrate for MMP-7 (PEG ${ }_{3400}-[\mathrm{Ahx}]-$ RPLALWRS-[Ahx]-PEG ${ }_{5000}$-K(5-FAM; PA construct) was obtained from Anaspec. This structure consists of a cleavable 8-mer peptide substrate (RPLALWRS) flanked by polyethylene glycol (PEG) groups of 3400 and $5000 \mathrm{~g} / \mathrm{gmol}$ and aminohexanoic acid (Ahx) groups to serve as spacer molecules, as well as a fluorescent tag (5-FAM) for cleavage detection. The N-terminus of the construct was a primary amine to facilitate further conjugation reactions. To stabilize the substrate and prevent additional modification, C-terminal amidation was performed during synthesis.

The N-terminal amine group of the substrate was conjugated to the surface of carboxylate QDs (CdSe dots coated with ZnS, 585 nm emission; Quantum Dot Corp., Hayward, CA) using 1-ethyl-3-(3-dimethylaminopropyl)carbodiimide (EDC). $250 \mu \mathrm{l}$ of QDs $(7.5 \mu \mathrm{M})$ was diluted to $1 \mu \mathrm{M}$ with $10 \mathrm{mM}$ borate buffer, $\mathrm{pH}$ 7.4. $80 \mu \mathrm{L}$ from a $10 \mathrm{mg} / \mathrm{mL}$ stock solution of the cleavable PA substrate was added to the solution, yielding a PA:QD molar ratio of 10.0:1. $57 \mu \mathrm{l}$ from a $10 \mathrm{mg} / \mathrm{ml}$ stock solution of EDC was added and the mixture was stirred for $2 \mathrm{~h}$ at room temperature to complete the conjugation. Unconjugated PA construct was removed by performing three buffer exchanges in a 50,000 Da nominal molecular weight limit (NMWL) cutoff spin filter device (Amicon Ultra). The concentration of the final purified product was determined by measuring the absorbance at $575 \mathrm{~nm}$ using a QD extinction coefficient of $400,000 \mathrm{M}^{-1} \mathrm{~cm}^{-1}$.

\section{MMP-7 activity assay}

Studies were conducted to determine the susceptibility of both the PA and QD-PA constructs to exogenous MMP-7. Active MMP-7 (Calbiochem) was added to $100 \mu \mathrm{l}$ of a $50 \mathrm{nM}$ PA or QD-PA solution in borate buffer ( $\mathrm{pH}$ 7.4) supplemented with $50 \mu \mathrm{M} \mathrm{ZnSO}_{4}$. The total concentration of MMP-7 was varied in these samples from $100 \mathrm{nM}$ to $5 \mathrm{nM}$. Samples were then incubated at $37^{\circ} \mathrm{C}$ for various timepoints ranging from $1 \mathrm{~h}$ to $48 \mathrm{~h}$. Control studies involving QD-PA with no enzyme and with inactivated enzyme (by addition of $30 \mathrm{mM}$ EDTA) were carried out simultaneously for direct comparison. In the case of QD-PA constructs, any cleavage products were removed by spin filtration in a 50,000 NMWL cutoff device.

\section{Gel electrophoresis}

PA constructs were analyzed on a 10\%-20\% gradient tristricine polyacrylamide gel (Life Gels). Samples were loaded in a buffer containing $10 \%$ glycerol in tris-tricine running buffer (Life Gels). Gels were then run under a $150 \mathrm{mV}$ voltage for $\sim 1 \mathrm{~h}$. Control, unconjugated PA constructs were compared with cleaved PA constructs to straightforwardly visualize and confirm enzymatic activity against the substrate.

A quantitative determination of PA construct cleavage was made by digital analysis of gel electrophoresis images. Gel images were obtained as JPEG files and visually analyzed
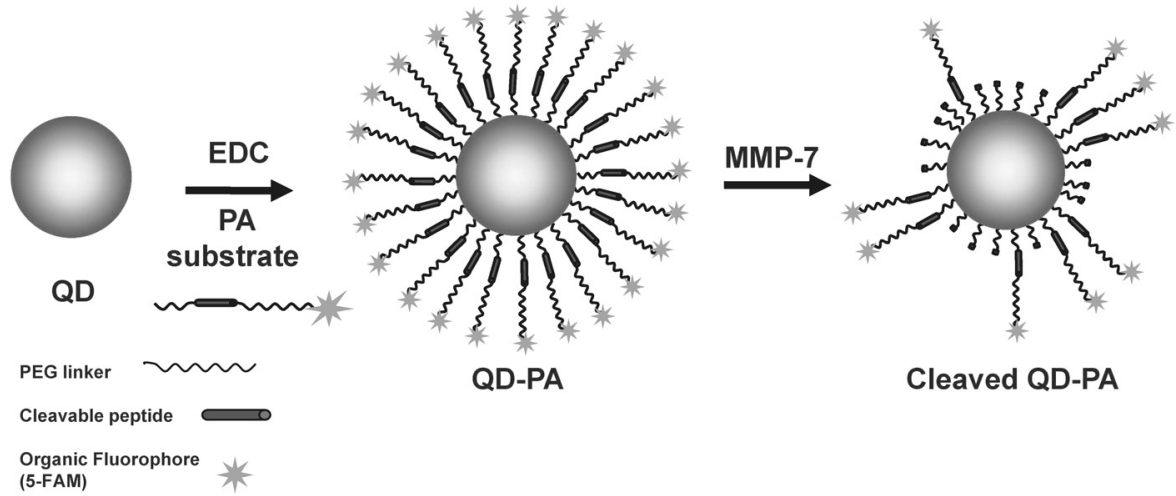

Cleaved QD-PA

Figure I Schematic of QD-PA synthesis and subsequent cleavage. 
in Doc-It LS software (UVP Inc.). Regions of interest were drawn around individual bands, and each band was electronically cut from the image and saved as a separate JPEG file. These files were loaded into a simple MATLab script designed to calculate quantitative image parameters. The composite fluorescence intensity for each band was calculated by correcting for background intensity and then summing the measured intensity of each pixel contained in the image. The relative percentage contained in each band was obtained by comparing its composite fluorescence to that of the total for the lane.

QD-PA constructs were assayed by loading samples directly on to a $0.8 \%$ bufferless agarose gel (E-Gel, Invitrogen Corp.). QDs subjected to the cleavage reaction were assayed against native carboxylate QDs, uncleaved QD-PA constructs, and QD-PA + EDTA. All gels were imaged for fluorescence on a BioDoc-It imaging system (UVP Inc.).

\section{Fluorescence spectroscopy}

Following PA conjugation and purification, the fluorescence spectra of QD-PA constructs were measured by fluorescence spectroscopy at $488 \mathrm{~nm}$ excitation (Perkin-Elmer LS 50B). Enzymatic cleavage of the QD-PA constructs was monitored by measuring changes to the fluorescence spectra following incubation with MMP-7. The spectra were scaled to normalize QD peak fluorescence $(-585 \mathrm{~nm})$ between all samples. The peak fluorescence intensity values at $\sim 519 \mathrm{~nm}$ and $\sim 585 \mathrm{~nm}$ were then directly compared to the spectra of control, uncleaved QD-PA particles to calculate the extent of PA construct cleavage following MMP-7 treatment.

\section{Size measurements}

Nanoparticle size was determined using dynamic light scattering (DLS) measurements. DLS measurements were conducted on a Malvern Nano Series Zetasizer with a $633 \mathrm{~nm}$ laser. The duration of the scans was $60 \mathrm{~s}$, and 3 scans were accumulated. The concentration of all QD samples was $0.25 \mu \mathrm{M}$.

\section{Statistics}

Statistical significance of experimental populations was assessed using an upaired t-test and determined by SigmaStat (Jandel Scientific Software). Differences were termed significant for $\mathrm{p}<0.05$.

\section{Results and discussion In vitro cleavage of the PA construct}

The PA construct is highly susceptible to cleavage by MMP-7. Prior to conjugation to the QD surface, PA constructs were assayed for susceptibility to enzymatic activity. Analysis of gel electrophoresis images of unconjugated PA (prior to conjugation to the NP surface) confirms specific cleavage of these constructs by MMP-7 (Figure 2). Lanes 1 and 2 confirm the presence of the intact, fluorescently labeled PA structure. These bright, low mobility bands are nearly extinguished after $24 \mathrm{~h}$ exposure to MMP-7. This cleavage also results in the generation of a very bright, high mobility band corresponding to the low molecular weight, labeled cleavage product (Figure 2, lanes 3 and 4). PA construct cleavage was completely abolished by the addition of $30 \mathrm{mM}$ EDTA (a zinc chelator; Figure 3, lanes 5 and 6), as the band corresponding to

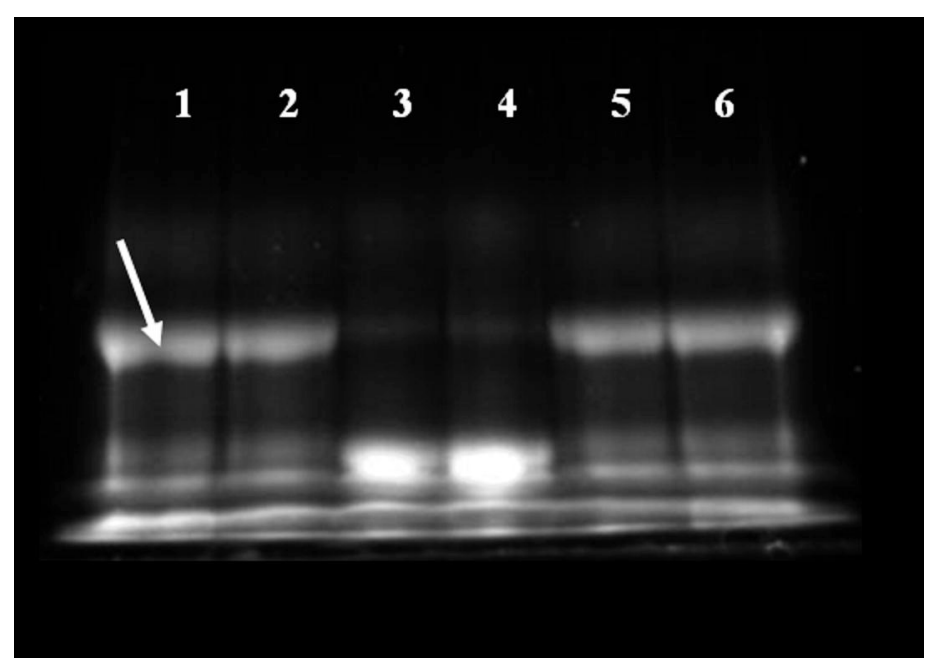

Figure 2 Gel electrophoresis confirms cleavage of the soluble, unconjugated PA construct by MMP-7. Lanes I and 2 show the unconjugated PA construct prior to enzymatic exposure as a control. The bright, low mobility band (identified by the white arrow) represents the intact high molecular weight PA structure. Lanes 3 and 4 represent the PA construct exposed to MMP-7 for 24 hours, while lanes 5 and 6 show the inhibition of MMP-7 activity by EDTA. 
the cleaved PA construct is absent in these wells. These results are consistent with the near complete proteolysis of the PA construct at the site of the MMP target peptide and inhibition of the zinc-dependent MMP-7 enzyme by EDTA.

A quantitative determination of PA construct cleavage was made by digital analysis of gel electrophoresis images. From this analysis, the percentage of PA construct contained in each sample was determined. Control, uncleaved soluble PA construct contains $35.6 \pm 3.4 \%$ intact PA structure, as determined from 4 independent trials. The remaining $64.4 \%$ of the fluorescence intensity in these bands represents high mobility structures consistent with 5-FAM labeled reactants and intermediates. $4.6 \pm 0.7 \%$ of the intact PA structure remains after exposure to $100 \mathrm{nM}$ MMP-7 for 24 hours. Therefore, $87.2 \pm 0.7 \%$ of the initial intact PA construct was cleaved $(\mathrm{p}<0.001)$ and $12.8 \pm 0.7 \%$ remained intact

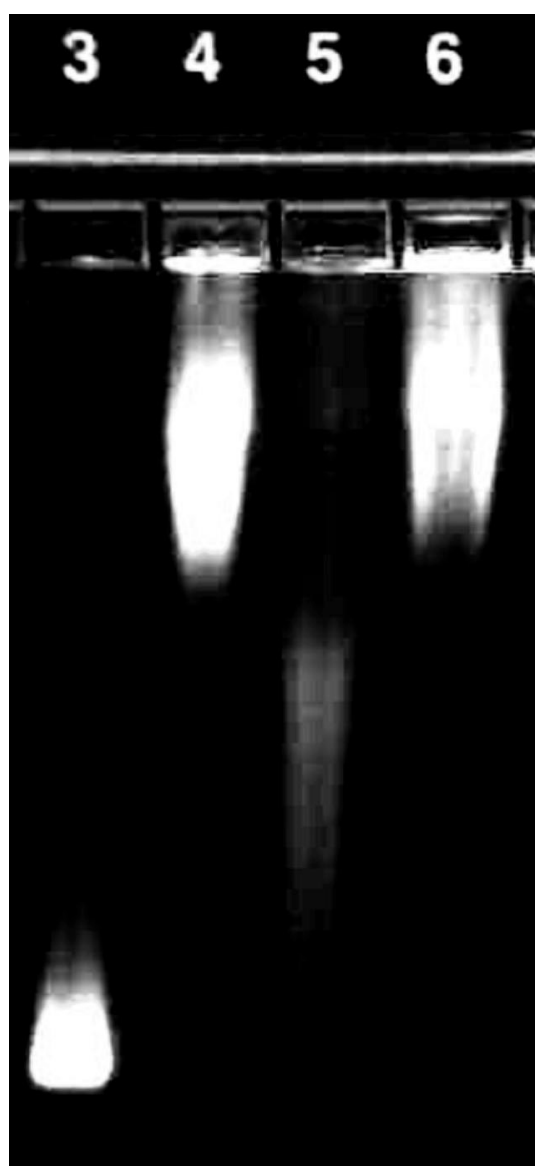

Figure 3 The surface of carboxylate QDs was successfully functionalized with the cleavable PA construct. Mobility of QDs modified with the cleavable PA construct (lane 4, diffuse band) is reduced compared with that of unmodified QDs (lane 3). $24 \mathrm{~h}$ exposure of the QD-PA conjugates to MMP-7 (lane 5) leads to a partial recovery of mobility, Increased mobility after exposure to MMP-7 can be completely inhibited by the addition of $30 \mathrm{mM}$ EDTA (lane 6). This image is representative of three independent trials. after enzymatic exposure, confirming the high susceptibility of the PA construct to MMP-7 proteolysis. Samples treated with MMP-7 + $30 \mathrm{mM}$ EDTA contain $35.5 \pm 2.0 \%$ intact PA structure, a value equivalent to the amount measured in control samples in the absence of MMP-7 ( $p=0.966)$.

\section{QD-PA construct synthesis and activity}

The surface of carboxylate QDs was successfully modified with the PA construct. This was evident as the size of the nanoparticle increased after functionalization. The size of the unmodified QD is $18.2 \pm 0.3 \mathrm{~nm}$, according to dynamic light scattering (DLS) measurements. After functionalization, the size of the quantum dot functionalized with the PA construct (QD-PA) is $81.8 \pm 10.5 \mathrm{~nm}$. Additionally, agarose gel electrophoresis shown in Figure 3 reveals that conjugation of the PA construct to the QD surface results in a significant decrease in electrophoretic mobility (lane 4) over unmodified carboxylate QDs (lane 3), presumably corresponding to an increase in MW and surface charge characteristics of the QD following PA conjugation. MMP-7 treatment results in partial recovery of mobility (lane 5), consistent with a reduction of surface-associated molecular mass mediated by proteolytic cleavage. Mobility recovery is not complete, consistent with persistence of the proximal portion of the PA construct on the QD surface after proteolytic cleavage. MMP-7 mediated increase in mobility is reversed with the addition of $30 \mathrm{mM}$ EDTA during cleavage (lane 6), suggesting inhibition of MMP-7 activity by EDTA. Changes in relative mobilities provide evidence of successful QD surface functionalization, subsequent proteolytic degradation, and inhibition of enzyme activity expected in this system.

QD-PA synthesis and activity was further confirmed by measuring the fluorescence spectra of the resulting conjugates. Following conjugation and purification, the fluorescence spectra of the QD-PA constructs were measured (Figure 4). These spectra reveal fluorescence maxima at approximately 520 and $585 \mathrm{~nm}$, corresponding to the emission maxima of the 5-FAM labeled PA construct (519 nm) and of the QD base (585 $\mathrm{nm})$, respectively.

Enzymatic cleavage was monitored by measuring changes to the fluorescence spectra of the QD-PA constructs following incubation with MMP-7 (Figure 4, QD-PA + $100 \mathrm{nM}$ MMP-7). Relative to control, uncleaved QD-PA constructs, these spectra reveal a reduced fluorescence peak at $519 \mathrm{~nm}$, suggesting loss of 5-FAM fluorescence from the QD-PA construct, consistent with proteolysis of the PA construct at the site of the MMP-7 target peptide and subsequent removal of cleavage products. This result is consistent with gel 


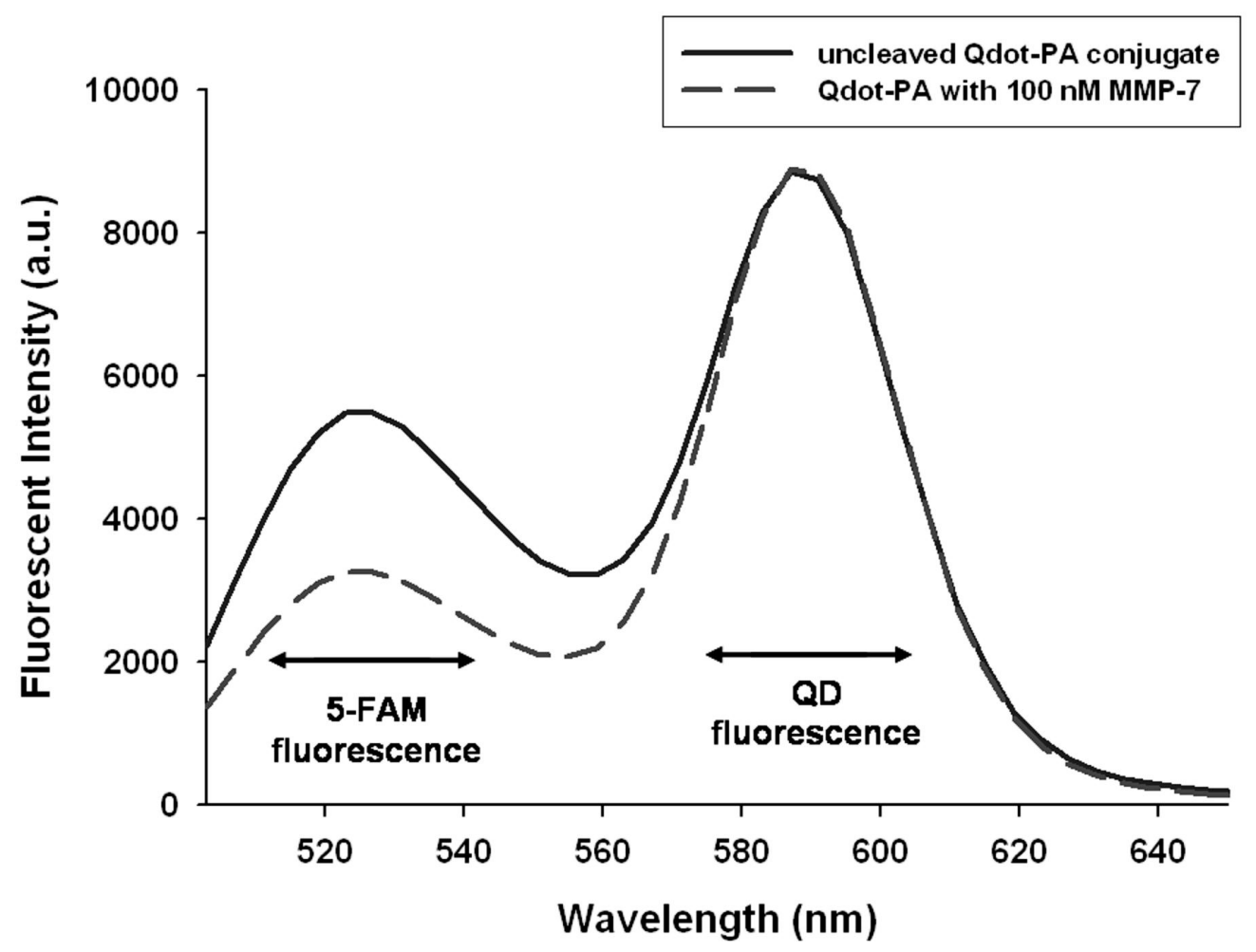

Figure 4 QDs modified with PA construct possess two fluorescence emission peaks consistent with colocalized QD and PA construct fluorescence. Exposure to MMP-7 (dashed line) results in reduced 5-FAM fluorescence intensity at approximately $520 \mathrm{~nm}$ when compared to control, uncleaved QD-PA samples (solid line). Results shown are representative of $\mathrm{n} \geq 3$ trials for all experimental groups.

electrophoresis images and computed results, demonstrating retention of PA proteolytic susceptibility following conjugation to the QD surface. Additional studies revealed no significant photobleaching of either 5-FAM or QD over the course of the measurements. Furthermore, no significant change in QD fluorescence intensity (at $585 \mathrm{~nm}$ ) was observed upon PA cleavage from the surface.

Fluorescence measurements were used to produce a quantitative analysis of PA construct cleavage. These measurements (Figure 5) confirm significant cleavage of the PA construct from the QD surface. Cleavage increased steadily with incubation time, reaching a plateau after $24 \mathrm{~h}(90.9 \pm$ $15.4 \%$ cleavage), consistent with cleavage of unconjugated PA constructs under the same conditions $(87.1 \pm 0.7 \%$ cleavage). Measured cleavage values are highly dependent on incubation time, following an exponential rise to maximum curve describing traditional enzyme kinetics.

Cleavage of the PA construct from the QD surface was also influenced by initial MMP-7 concentration. Cleavage was measured from samples using MMP-7 concentrations ranging from $100 \mathrm{nM}$ to $5 \mathrm{nM}$ (reflecting the range of expected concentrations in vivo) as shown in Figure 6. Although the cleavage response measured for each MMP-7 concentration follows the same general trend, the extent of cleavage is markedly altered, declining as MMP-7 concentration was reduced. Cleavage at early timepoints $(\mathrm{t} \leq 6 \mathrm{~h})$ is insensitive to MMP-7 concentrations above approximately $10 \mathrm{nM}$.

Previous literature reports the $\mathrm{K}_{\mathrm{m}}$ of unmodified PA substrate as $26 \mu \mathrm{M}$ (Welch et al 1995). Thus, at substrate concentrations below this value, PA cleavage is expected to be independent of MMP-7 concentration. Bulk PA concentrations used in the current study range from 37.5-100 nM, well below the reported $\mathrm{K}_{\mathrm{m}}$. However, at the given substrate concentration, and at low MMP-7 concentrations (eg, $5 \mathrm{nM}$ and $10 \mathrm{nM}$ ), MMP-7 is the limiting reagent. At higher MMP7 concentrations (eg, $100 \mathrm{nM}$ ), MMP-7 concentration meets or exceeds substrate concentration. Thus, cleavage behavior transitions from a regime where MMP-7 is the limiting component to a regime where PA is the limiting component, resulting in measurements consistent with conventional enzyme kinetics.

Literature has shown that MMP-7 concentration can vary widely, with overexpression noted in various tumor phenotypes (McIntyre et al 2004). While few quantitative measures of MMP-7 concentration have been performed, 


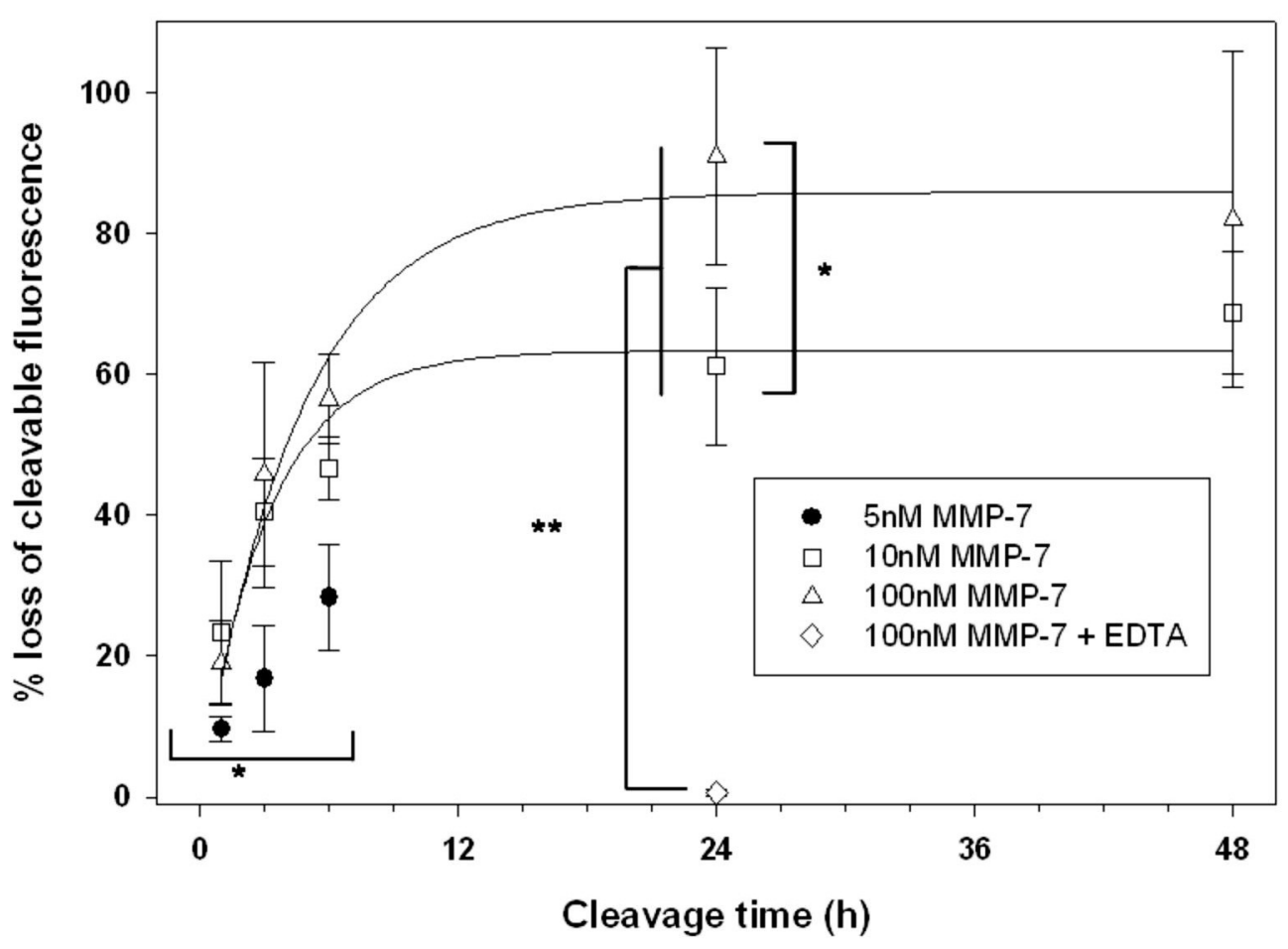

Figure 5 Fluorescence spectra of control, uncleaved QD-PA structures were compared to those of samples incubated with variable concentrations of MMP-7. Statistical measurements: ${ }^{*}=\mathrm{p}<0.05,{ }^{*} \mathrm{p}<0.00 \mathrm{I} . \mathrm{n} \geq 3$ for all concentrations and timepoints.

previous studies have utilized concentrations of up to $100 \mathrm{nM}$ in vitro (Kioi et al 2003; Wang et al 2005), while others have measured comparable concentrations of additional MMPs in vivo (Furuya et al 2000). This evidence suggests likely local MMP-7 concentrations in tumor of at least $50 \mathrm{nM}$, corresponding with the levels utilized in the current study. Furthermore, our studies reveal that proteolytic susceptibility of QD-PA probes saturates at MMP-7 concentrations above $10 \mathrm{nM}$. Thus, at enzyme concentrations at or above this threshold, QD-PA probes are expected to exhibit maximal activity.

As particle activation time and tissue residence time (ie, particle velocity) are competing factors, this ratio is an important consideration with potential for significant impact on in vivo utility of QD-PA constructs. If activation time is long compared to residence time (eg, for vascular targets), then the NP will presumably be transported away during activation and before specific interaction can occur. In this case, PA delivery has characteristics similar to those for current targeted approaches. However, the small size and surface passivation (PEGylation) of the QD-PA construct combine to create a construct likely capable of extravasation and tumor accumulation, enabling access to targets within the tissue. In this compartment, particle transport is typically very slow, allowing ample time for particle activation. At the predicted tissue MMP-7 concentrations, QD-PA probes require $<6 \mathrm{~h}$ for activation (defined as $50 \%$ cleavage), with significant activity reached within $3 \mathrm{~h}$. This activation time relates well to NP tissue residence times (Kaul and Amiji 2004; Montet et al 2006), yielding probe performance characteristics consistent with in vivo triggered targeting.

\section{Conclusions}

The QD-PA constructs described here could potentially provide a powerful, versatile platform for targeted delivery and biosensing for both in vitro and in vivo applications. Although the importance of therapeutic targeting has been acknowledged for more than two decades, few viable options for targeted therapy have been successfully implemented. Many treatment regimens continue to rely on systemic administration that compromises drug concentrations delivered to target tissue based on toxicity resulting from specific and nonspecific delivery to nontarget cells (Frei 1997).

The modular structure of the QD-PA constructs provides multiple parameters for performance optimization, with various factors presumably modulating the overall activity of the construct. Successful optimization requires consideration of the nanoscale, biological chemistry, and 


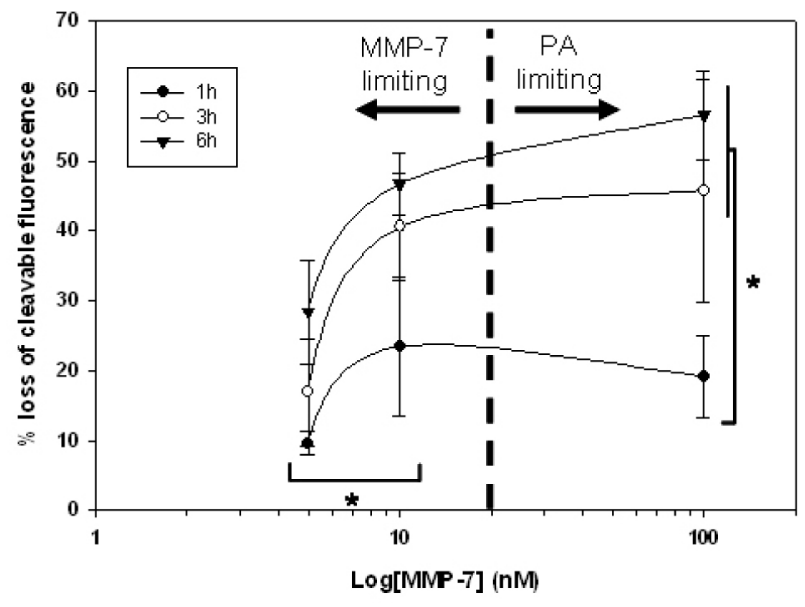

Figure 6 Cleavage of PA construct from the QD surface at early timepoints $(\mathrm{t}<6 \mathrm{~h})$ is modulated by MMP-7 concentration. Quantitative cleavage measurements were analyzed in the form of a dose response curve to highlight the influence of enzyme concentration on construct cleavage. ${ }^{*}=p<0.05, n \geq 3$ for all MMP-7 concentrations and timepoints.

physiological transport of the overall construct. PEG length is one variable with potential to significantly affect multiple characteristics including enzyme susceptibility (by limiting enzyme access to the substrate), nonspecific binding limitation, effective ligand concealment (in future structures), and particle mobility. Additional structural parameters including enzyme substrate configuration, ligand selection, and QD ligand surface density (number of molecules conjugated to the QD surface) can also directly affect probe performance. Tailoring of these multiple characteristics should enable optimization of probe performance for the requirements of both in vitro and in vivo systems.

PA targeting requires the colocalization of the cleavable mask and the targeting ligand, necessitating a particulate approach. In the exemplar described here, the NP is a QD, used to provide a fluorescent beacon for performance assessment. Subsequent work will employ this construct, amended to include a targeting ligand, to assess proteolytically activated targeting in vitro and in vivo by quantitative fluorescence measurements and fluorescence imaging. While the appropriateness of QDs for clinical applications remains theoretically limited due to their heavy metal content, the PA targeting approach described here can be applied to other nanostructures approved for use in humans. These nanomaterials include iron oxide nanoparticles for magnetic resonance image contrast enhancement and drug delivery structures such as polymers, liposomes, polymersomes, and gene delivery vehicles.

\section{Acknowledgments}

The authors gratefully acknowledge the Department of Defense Idea Award BC043908 for funding.

\section{References}

Adachi Y, Yamamoto H, Itoh F, et al. 2001. Clinicopathologic and prognostic significance of matrilysin expression at the invasive front in human colorectal cancers. Int J Cancer, 95:290-4.

Akerman ME, Chan WC, Laakkonen P, et al. 2002. Nanocrystal targeting in vivo. Proc Natl Acad Sci USA, 99:12617-21.

Ballou B, Lagerholm BC, Ernst LA, et al. 2004. Noninvasive imaging of quantum dots in mice. Bioconjug Chem, 15:79-86.

Bogenrieder T, Herlyn M. 2003. Axis of evil: molecular mechanisms of cancer metastasis. Oncogene, 22:6524-36.

Bremer C, Tung CH, Weissleder R. 2001. In vivo molecular target assessment of matrix metalloproteinase inhibition. Nat Med, 7:743-8.

Bremer C, Tung CH, Weissleder R. 2002. Molecular imaging of MMP expression and therapeutic MMP inhibition. Acad Radiol, 9(Suppl 2):S314-15.

Crawford HC, Scoggins CR, Washington MK, et al. 2002. Matrix metalloproteinase-7 is expressed by pancreatic cancer precursors and regulates acinar-to-ductal metaplasia in exocrine pancreas. $J$ Clin Invest, 109:1437-44.

Frei E III, Antman KH. 1997. Combination chemotherapy, dose and schedule. In: Holland JF, Frei E III, Bast RC, et al. eds. Cancer Medicine, 4th edn Williams and Wilkins: Philadelphia, 817-37.

Furuya M, Ishikura H, Ogawa Y, et al. 2000. Analyses of matrix metalloproteinases and their inhibitors in cyst fluid of serous ovarian tumors. Pathobiology, 68:239-44.

Gao X, Cui Y, Levenson RM, et al. 2004. In vivo cancer targeting and imaging with semiconductor quantum dots. Nat Biotechnol, 22:969-76.

Hallahan DE, Geng L, Cmelak AJ, et al. 2001. Targeting drug delivery to radiation-induced neoantigens in tumor microvasculature. $J$ Control Release, 74(1-3):183-91.

Harris TJ, von Maltzahn G, Derfus AM, et al. 2006. Proteolytic actuation of nanoparticle self-assembly. Angew Chem Int Ed, 45:3161-5.

Hulboy DL, Gautam S, Fingleton B, et al. 2004. The influence of matrix metalloproteinase-7 on early mammary tumorigenesis in the multiple intestinal neoplasia mouse. Oncol Rep, 12:13-17.

Kaul G, Amiji M. 2004. Biodistribution and targeting potential of poly(ethylene glycol)-modified gelatin nanoparticles in subcutaneous murine tumor model. J Drug Target, 12(9-10):585-91.

Kim R, Toge T. 2004. Changes in therapy for solid tumors: potential for overcoming drug resistance in vivo with molecular targeting agents. Surg Today, 34:293-303.

Kioi M, Yamamoto K, Higashi S, et al. 2003. Matrilysin (MMP-7) induces homotypic adhesion of human colon cancer cells and enhances their metastatic potential in nude mouse model. Oncogene, 22:8662-70.

Kuhn SJ, Finch SK, Hallahan DE, et al. 2006. Proteolytic surface functionalization enhances in vitro magnetic nanoparticle mobility through extracellular matrix. Nano Lett, 6:306-12.

Kuhn SJ, Finch SK, Hallahan DE, et al. 2007. Facile production of multivalent enzyme-nanoparticle conjugates. Journal of Magnetism and Magnetic Materials, 311:68-72.

Liu C, Sun C, Huang H, et al. 2003. Overexpression of legumain in tumors is significant for invasion/metastasis and a candidate enzymatic target for prodrug therapy. Cancer Res, 63:2957-64.

Liu J, Zhang Q, Remsen EE, et al. 2001. Nanostructured materials designed for cell binding and transduction. Biomacromolecules, 2:362-8.

McIntyre JO, Fingleton B, Wells KS, et al. 2004. Development of a novel fluorogenic proteolytic beacon for in vivo detection and imaging of tumour-associated matrix metalloproteinase-7 activity. Biochem $J$, 377(Pt 3):617-28.

McIntyre JO, Matrisian LM. 2003. Molecular imaging of proteolytic activity in cancer. J Cell Biochem, 90:1087-97. 
Mitra A, Mulholland J, Nan A, et al. 2005. Targeting tumor angiogenic vasculature using polymer-RGD conjugates. J Control Release, 102:191-201.

Montet X, Montet-Abou K, Reynolds F, et al. 2006. Nanoparticle imaging of integrins on tumor cells. Neoplasia, 8:214-22.

Pastorino F, Brignole C, Marimpietri D, et al. 2003. Vascular damage and anti-angiogenic effects of tumor vessel-targeted liposomal chemotherapy. Cancer Res, 63:7400-9.

Phadtare S, Vinod VP, Mukhopadhyay K, et al. 2004. Immobilization and biocatalytic activity of fungal protease on gold nanoparticle-loaded zeolite microspheres. Biotechnol Bioeng, 85:629-37.

Rossi LM, Quach AD, Rosenzweig Z. 2004. Glucose oxidase-magnetite nanoparticle bioconjugate for glucose sensing. Anal Bioanal Chem, 380:606-13.

Sachdeva MS. 1998. Drug targeting systems for cancer chemotherapy. Expert Opin Investig Drugs, 7:1849-64.

Suzawa T, Nagamura S, Saito H, et al. 2002. Enhanced tumor cell selectivity of adriamycin-monoclonal antibody conjugate via a poly(ethylene glycol)-based cleavable linker. J Control Release, 79:229-42.

Suzawa T, Nagamura S, Saito H, et al. 2000. Synthesis and HPLC analysis of enzymatically cleavable linker consisting of poly(ethylene glycol) and dipeptide for the development of immunoconjugate. $J$ Control Release, 69:27-41.

Tang D, Yuan R, Chai Y, et al. 2005. Novel potentiometric immunosensor for determination of diphtheria antigen based on compound nanoparticles and bilayer two-dimensional sol-gel as matrices. Anal Bioanal Chem, 381:674-80.

Tung CH, Mahmood U, Bredow S, et al. 2000. In vivo imaging of proteolytic enzyme activity using a novel molecular reporter. Cancer Res, 60:4953-8.
Wang FQ, So J, Reierstad S, et al. 2005. Matrilysin (MMP-7) promotes invasion of ovarian cancer cells by activation of progelatinase. Int $J$ Cancer, 114:19-31.

Wang M, Sun C, Wang L, et al. 2003. Electrochemical detection of DNA immobilized on gold colloid particles modified self-assembled monolayer electrode with silver nanoparticle label. J Pharm Biomed Anal, 33:1117-25.

Weissleder R, Tung $\mathrm{CH}$, Mahmood U, et al. 1999. In vivo imaging of tumors with protease-activated near-infrared fluorescent probes. Nat Biotechnol, 17:375-8.

Welch AR, Holman CM, Browner MF, et al. 1995. Purification of human matrilysin produced in Escherichia coli and characterization using a new optimized fluorogenic peptide substrate. Arch Biochem Biophys, 324:59-64.

Wilson CL, Matrisian LM. 1996. Matrilysin: an epithelial matrix metalloproteinase with potentially novel functions. Int J Biochem Cell Biol, 28:123-36.

Yamamoto H, Adachi Y, Itoh F, et al. 1999. Association of matrilysin expression with recurrence and poor prognosis in human esophageal squamous cell carcinoma. Cancer Res, 59:3313-16.

Yamamoto H, Itoh F, Iku S, et al. 2001. Expression of matrix metalloproteinases and tissue inhibitors of metalloproteinases in human pancreatic adenocarcinomas: clinicopathologic and prognostic significance of matrilysin expression. J Clin Oncol, 19:1118-27.

Zhang D, Chen Y, Chen HY, et al. 2004. Silica-nanoparticle-based interface for the enhanced immobilization and sequence-specific detection of DNA. Anal Bioanal Chem, 379(7-8):1025-30.

Zhang Y, So MK, Rao J. 2006. Protease-modulated cellular uptake of quantum dots. Nano Lett, 6:1988-92.

Zucker S, Cao J. 2001. Imaging metalloproteinase activity in vivo. Nat Med, 7:655-6. 
